\title{
FORMULATION, OPTIMIZATION, AND IN VITRO CHARACTERIZATION OF DASATINIB LOADED POLYMERIC NANOCARRIERS TO EXTEND THE RELEASE OF THE MODEL DRUG
}

\author{
SANDEEP KUMAR REDDY ADENA ${ }^{1 *}$, KASI VISWANADH MATTE2 ${ }^{2}$, RAMOJI KOSURU ${ }^{3}$
}

1,2Department of Pharmaceutical Engineering and Technology, Indian Institute of Technology (BHU), Varanasi, India, ${ }^{3}$ Versiti Blood Research Institute, Milwaukee, USA

Email: ksandeep.rs.phe14@itbhu.ac.in

Received: 07 May 2021, Revised and Accepted: 21 Jun 2021

\begin{abstract}
Objective: The present research aims to design, develop, optimize, characterize and evaluate dasatinib (DSB) loaded polymeric nanocarriers to treat chronic myeloid leukaemia (CML) by adopting a quality by design (QbD) approach.

Methods: Risk assessment was performed by using failure modes and effects analysis, and optimization of nanoformulation was done by adopting $2^{3}$ full factorial design. The optimized nanoformulation was characterized by different characterization techniques and evaluated by various in vitro studies.

Results: Surface morphology and shape were found to be smooth and spherical. Stability study results revealed that the nanoformulation could be stored in all three storage conditions for safe and long-term use since it retained its pharmaceutical properties. Drug release was $32.06 \%$ in the first $4 \mathrm{~h}$ and $79.34 \%$ by the end of $48 \mathrm{~h}$ which infers a sustained-release pattern. The hemocompatibility results showed no sign of hemolysis. Cellular uptake study showed approximately 10 to 20 -fold much higher intracellular fluorescence intensities of nanoformulation than DSB. Cytotoxicity results confirmed that when compared to the pure drug, the optimized nanoformulation have a potential cytotoxic effect in the treatment of CML since it exhibited a significantly more \% growth inhibition. Cell apoptosis assay revealed that the nanoformulation could provide significant antileukaemia activity against K562 cells and further induce K562 cell death with a dose and time-dependent manner.
\end{abstract}

Conclusion: The results of the characterization and evaluation studies showed that the developed nanoformulation offered significant advantages, making it a potential delivery system of DSB for more effective treatment of CML.

Keywords: Quality by design, Gold nanoparticles, Dasatinib, Full factorial design, Sustained release

(C) 2021 The Authors. Published by Innovare Academic Sciences Pvt Ltd. This is an open access article under the CC BY license (https://creativecommons.org/licenses/by/4.0/) DOI: https://dx.doi.org/10.22159/ijap.2021v13i5.41995. Journal homepage: https://innovareacademics.in/journals/index.php/ijap

\section{INTRODUCTION}

Dasatinib (DSB) is a novel, potent and multi-targeted inhibitor of BCR-ABL and Src tyrosine kinases activity in the leukaemia cells approved by US FDA (United States Food and Drug Administration) $[1,2]$. For newly diagnosed patients, tyrosine kinase inhibitor therapy by DSB is the first-line treatment. But the treatment with DSB is associated with hematological and non-hematological adverse effects [3]. These adverse effects are likely because of endothelial hyperpermeability, and the interaction of DSB with nontumor-related cells and processes leads to a reduction in the dose or discontinuation of the treatment [4]. DSB is a biopharmaceutics classification system (BCS) class-II drug, possessing high permeability and low solubility. It has low bioavailability, shorter plasma half-life, lack of specificity, early degradation, rapid elimination, and is recommended in high dose. All these characteristics associated with DSB may be efficiently eradicated by using gold nanoparticles (GNPs) as a drug carrier [5, 6].

Gold nanoparticles (GNPs) have turned out to be an exceptional drug nanocarrier for anti-cancer drugs as they can effectively carry and protect a high therapeutic payload and selectively delivers the drug at the tumor site by active/passive targeting methods thereby enhancing the therapeutic index [7]. They have turn out to be a potential carrier for targeted drug delivery because of its biodegradability, biocompatibility, and bioavailability [8]. GNPs can be engineered in different approaches to sense a stimulus and instantly act to the response by releasing the drug payload at a specific site into the cells or tissue [9]. The significant advantages of the GNPs include reduced dosage, controlled biodistribution, modulated pharmacokinetics, reduced toxicity, and most importantly, improved patient compliance $[10,11]$.

Reduction of the Au (III) ions to their metallic form is the most significant step in the synthesis of GNPs. It is usually carried out by using different chemical reducing agents by chemical reduction method, but its use affects the purity of the obtained GNPs and are also harmful to human beings due to their biocompatibility and longterm environmental sustainability issues $[12,13]$. To overcome all these constraints, green reduction methods have been developed alternatively. A natural reducing agent, Chitosan (Ch), is widely used for the development of GNPs because of its non-toxic, biocompatible, cost-effective, eco-friendly, large-scale production suitability, sustained release and tumor-inhibiting properties $[14,15]$. Usually, the synthesized Ch-GNPs are extremely biocompatible with pharmaceutical and biomedical applications and possesses unique bioactivities (antitumor, antiviral and antimicrobial) [16].

The stabilization of colloidal GNPs by preventing flocculation is one of the most significant and essential features and enables extensive physical and biological applications [17]. Biodegradable polymers are approved by the US FDA in the development of nanoparticles and are most commonly used for the stabilization of GNPs and improving its dispersity [18]. Among them, poly lactic-co-glycolic acid (PLGA) is used widely mainly because it is biocompatible, and its degradation rates can be tailored to release the encapsulated payload for a prolonged time. PLGA is a safely administrable polymer and the best candidate for a sustained-release drug delivery system [19].

In the development of DSB-PLGA-Ch-GNPs, QbD (Quality by Design) is employed, which is a proactive, modern and scientific approach to product design and development. It enhances the process capability, formulation design, reduces product variability and involves a systematic approach to make sure the quality of the final product. It is a very efficient way to enhance the value of research and minimize the development time and cost. QbD develops a thorough understanding of the compatibility of the final product to all of its critical process and formulation attributes that are involved in product development. It helps to identify the root cause of a quality issue by efficient analysis and provides insights throughout the process of product development and aims at achieving the desired quality product with anticipated and predetermined specifications [20-22]. 
The aim of the present research was to synthesize and stabilize potent, non-toxic, cost-effective, eco-friendly, selectively targeted, sustained-release drug-loaded gold nanocarriers by green reduction method using minimum raw materials and time, and preserving its stability and bioactivity during fabrication and release. DSB-PLGACh-GNPs were optimized by QbD approach. Physicochemical and morphological characterization of the optimized DSB-PLGA-Ch-GNPs was done by DLS (dynamic light scattering), LDV (laser doppler velocimetry), FTIR (Fourier transform infrared spectroscopy), XRD (x-ray diffraction), EDXS (energy dispersive x-ray spectroscopy), TEM (transmission electron microscopy), SAED (selected area electron diffraction), and AFM (atomic force microscopy). Further, the optimized DSB-PLGA-Ch-GNPs were evaluated for stability at different storage conditions, in vitro drug release and release kinetics, in vitro hemocompatibility, cellular uptake by confocal fluorescence microscopy, in vitro cytotoxicity by sulforhodamine B (SRB) assay in K562 cell lines, and cell apoptosis by flow cytometry in order to test and provide evidence for the developed DSB-PLGACh-GNPs to be used as a potential delivery system of DSB for more effective treatment of CML.

\section{MATERIALS AND METHODS}

\section{Materials}

Dasatinib was provided as a gift sample by MSN Laboratories Pvt. Ltd., Hyderabad, India. Gold chloride hydrate and chitosan were purchased from Sigma-Aldrich, Mumbai, India; DMSO (dimethyl sulfoxide) was purchased from Thermo Fisher, Mumbai, India; PLGA was purchased from Evonik Industries Pvt. Ltd., Mumbai, India; dialysis membrane was purchased from HiMedia, Mumbai, India; methanol and acetonitrile of HPLC grade was purchased from Merck Ltd., Mumbai, India; and all other chemicals used in the present investigation from S D Fine Chem Ltd., Mumbai, India. Ultrapure milli-Q water was used all over the investigation.

\section{Methods}

\section{Formulation of DSB-PLGA-Ch-GNPs}

GNPs were synthesized by adding chitosan to the gold chloride hydrate aqueous solution, and the resulting dispersion was placed on the magnetic stirrer (IKA, Germany) at $70{ }^{\circ} \mathrm{C}$ until it changes to a deep ruby red color. Ch-GNPs formation was confirmed with UVVisible spectroscopy (UV-1800, Shimadzu, Japan) by recording a peak maximum at $525 \mathrm{~nm}$. The synthesized Ch-GNPs were centrifuged (RC 4100F, Eltek, India) at 12,000 rpm for $30 \mathrm{~min}$, washed thoroughly with deionized water to remove excess chitosan and gold. Finally, to remove any ionic impurities present in the $\mathrm{Ch}$ GNPs dispersion, it was dialyzed using a dialysis tube for $12 \mathrm{~h}$.

DSB-PLGA-Ch-GNPs were prepared by adding PLGA to Ch-GNPs dispersion and stirred for $24 \mathrm{~h}$. To the resulting dispersion, DSB in DMSO was added and subjected to homogenization (Ika T25 Ultra Turrax, Germany) for $2 \mathrm{~h}$, followed by sonication (Q Sonica Q 500 , Newtown, USA) for $15 \mathrm{~min}$. To allow proper interaction, the resulting dispersion was stored at room temperature for $12 \mathrm{~h}$, followed by stirring for $6 \mathrm{~h}$. Further, the resulting dispersion was subjected to centrifugation (Remi, Vasai, India) for $30 \mathrm{~min}$ at 10,000 rpm, followed by freeze-drying (Labconco, Kansas, USA). The obtained lyophilized DSB-PLGA-Ch-GNPs were stored in a desiccator until further use.

Table 1: Prioritization of critical quality attributes based on failure modes and effects analysis

\begin{tabular}{llll}
\hline CQAs (Factors) & Responses & \\
\cline { 2 - 4 } & Particle size & \% Entrapment efficiency & Zeta potential \\
\hline Polymer type & Low & Low & Low \\
Polymer concentration & High & High & Medium \\
Stirring speed & Medium & Low & Low \\
Stirring time & High & High & Medium \\
Solvent type & Low & Low & Low \\
Solvent ratio & Low & Low & Low \\
Sonication frequency & Medium & Medium & Low \\
Sonication time & High & Migh & Medium \\
Temperature & Low & Medium & Low \\
Centrifugation speed & Low & Medium & Low \\
Centrifugation time & Low & Medium & \\
\hline
\end{tabular}

\section{Risk assessment studies}

Risk is an amalgamation of the likelihood of incidence of damage and the intensity of that damage. Each and every component that is used in the development of the pharmaceutical product exhibits some risk. To reduce the risk and avoid potential product failure, risk assessment is performed. It helps to identify the critical quality attributes (CQAs) that may affect the product's final quality and raise the quality of process or method. Failure modes and effects analysis (FMEA) is an important tool to identify all the critical potential risks based on rank modes of relative usefulness to prioritize the CQAs as low, medium and high [23]. Table 1 shows the prioritization levels of the factors ahead of employing the design of experiments to optimize the manufacturing process of DSB-PLGA-Ch-GNPs.

\section{Risk assessment screening}

PBD (Plackett-Burman design) was employed to screen and evaluate the significant risk factors that may influence the identified CQAs. The preliminary screening experiments with factors and the CQAs were evaluated and coded as "+" and "-" for high levels and low levels respectively for simplification. PBD was constructed with 8 factors, 3 responses and 12 experiments using Minitab 17 software (Minitab Inc., Pennsylvania, USA), and statistical analysis was performed. ANOVA was employed to test the significance of PBD and coefficients for each factor. Further, Pareto chart was employed to choose the main factors that have a significant effect on CQAs (responses).

\section{$2^{3}$ full factorial design (FFD) for optimization of DSB-PLGA-Ch- GNPs}

The effects of the three main factors (polymer concentration (PC), sonication time (SoT) and stirring time (ST)) were tested on the responses (particle size (PS), \% entrapment efficiency (\% EE), and zeta potential (ZP). By the preliminary screening, $2^{3}$ FFD (3-factor, 2level) was applied to optimize the DSB-PLGA-Ch-GNPs. FFD was selected for the present study as it generates fewer experimental runs with three factors, and also it is appropriate for exploring the quadratic equation, deriving the polynomial equation and plotting response surface plots. The experimental design was constructed and evaluated by using Minitab 17 software. Eight batches were prepared as per FFD, and ANOVA was employed to establish the significance of the experimental model. The polynomial equation generated by the FFD elucidating the effect of factors on each of the responses is as follows:

$$
\mathrm{R}_{\mathrm{i}}=\alpha_{0+} \alpha_{1} \mathrm{X}_{+} \alpha_{2} \mathrm{Y}_{+} \alpha_{3} \mathrm{Z}+\alpha_{12} \mathrm{XY}+\alpha_{13} \mathrm{XZ}+\alpha_{23} \mathrm{YZ}_{+} \alpha_{123} \mathrm{XYZ}
$$

Where $R_{i}$ is the measured response, $\alpha_{0}$ is the intercept, $\alpha_{1}$ to $\alpha_{123}$ is the associated coefficients coded values of the responses. X, Y, and Z are the coded value of the factors. 
Physicochemical and morphological characterization of optimized DSB-PLGA-Ch-GNPs

\section{Determination of PS and ZP}

The PS and size distribution of DSB-PLGA-Ch-GNPs were determined by DLS. It provides an accurate measure of nanoparticles hydrodynamic size. DSB-PLGA-Ch-GNPs samples were diluted by using ultrapure milli- $Q$ water to get a concentration close to 100 $\mu \mathrm{g} / \mathrm{ml}$ and filtered through $0.45 \mu \mathrm{m}$ membrane syringe filter before loading the samples into the cuvette. The sample solution was illuminated, and its scattering intensity was recorded at a fixed scattering angle with a photon detector [24].

The ZP of DSB-PLGA-Ch-GNPs was determined by LDV in which an electrical field is applied across the DSB-PLGA-Ch-GNPs sample, and the electrophoretic mobility of nanoparticles was measured [25]. Desla ${ }^{\mathrm{TM}}$ Nano Common (Beckman Coulter ${ }^{\circledR}$ Instruments, UK) was used to measure the PS and ZP of the DSB-PLGA-Ch-GNPs.

\section{Determination of $\% \mathrm{EE}$}

The \% EE of DSB-PLGA-Ch-GNPs was determined by the centrifugation method by separating the supernatant free drug content. The nanoparticles were centrifuged for $30 \mathrm{~min}$ at 12,000 rpm in order to separate the unentrapped drug from the DSB-PLGACh-GNPs. After centrifugation, the supernatant solution containing the free drug was separated, and pH 7.4 PBS (phosphate-buffered saline) was added and vortexed for 5 min. The resultant solution was subjected to filtration through a $0.22 \mu \mathrm{m}$ filter, and the filtrate was analyzed using a UV-Visible absorption spectrophotometer [26]. The $\% \mathrm{EE}$ was calculated by using the formula:

$$
\% \mathrm{EE}=\frac{\text { Total amount of drug added-Amount of free drug in the supernatant }}{\text { Total amount of drug added }} \times 100
$$

\section{FTIR}

FTIR is primarily employed to know the presence of certain functional groups in the molecule. FTIR spectrophotometer (FTIR8400S, Shimadzu, Japan) instrument was used to obtain the FTIR spectra. To obtain the spectra DSB, PLGA, Ch, Ch-GNPs and DSBPLGA-Ch-GNPs samples were mixed individually with $\mathrm{KBr}$. In a manual press, the samples were compressed separately into a very thin disc, and the \% transmittance $(\% \mathrm{~T})$ of the samples was recorded in the spectral region of $4000-500 \mathrm{~cm}^{-1}$ in diffuse reflectance mode [27].

\section{XRD}

It was used to examine the physical properties of the drug and excipients in pure form and in the nanoformulation, to check the crystallinity and any alteration in the drug's physical state after the entrapment. The XRD spectra of optimized nanoformulation, DSB, PLGA, Ch, and GNPs were obtained by using x-ray diffractometer (Rigaku, Japan). The diffractograms were recorded by using Nifiltered $\mathrm{Cu} \mathrm{K} \alpha 1 \mathrm{x}$-ray radiation source, $15 \mathrm{~mA}$ amperage, $40 \mathrm{kV}$ tube voltage, $10^{\circ} \mathrm{min}^{-1} \mathrm{scan}$ speed, $0.02^{\circ}$ step width and $20^{\circ}$ to $80^{\circ} 2 \theta$ scanning range [28].

\section{EDXS}

EDXS analysis was done to establish the elemental composition of the optimized DSB-PLGA-Ch-GNPs. EDAX (Ametek, NJ, USA) instrument was used to acquire the EDXS spectrum. The EDXS spectrum was recorded by focusing DSB-PLGA-Ch-GNPs at a densely occupied region [29].

\section{TEM and SAED}

TEM was employed to examine the size, shape, and dispersity of the optimized DSB-PLGA-Ch-GNPs. The sample was prepared by placing a tiny DSB-PLGA-Ch-GNPs dispersion drop on a carbon-coated copper grid. The sample was allowed to dry at room temperature. FEI Tecnai G 20 X-TWIN instrument was used to acquire the TEM micrographs of DSB-PLGA-Ch-GNPs [30]. SAED was employed in conjunction with TEM, to check out the amorphous nature of the optimized DSB-PLGA-Ch-GNPs [29].

\section{AFM}

AFM was employed to explore the surface morphology of the optimized DSB-PLGA-Ch-GNPs. The sample was prepared by placing a tiny drop of DSB-PLGA-Ch-GNPs dispersion on the mica surface. It was allowed to dry at room temperature to form a very thin film. 3D and 2D images of DSB-PLGA-Ch-GNPs were acquired by using an atomic force microscope (NT-MDT, NTEGRA Probe Nanolaboratory, Russia) [31].

\section{Stability study}

To evaluate the effect of stress conditions, optimized DSB-PLGA-ChGNPs were subjected to refrigerated $\left(4 \pm 1{ }^{\circ} \mathrm{C}\right)$, room temperature $\left(25 \pm 2{ }^{\circ} \mathrm{C}\right)$ and accelerated $\left(40 \pm 2{ }^{\circ} \mathrm{C} / 75 \pm 5 \% \mathrm{RH}\right)$ conditions as per ICH guidelines. Freshly prepared DSB-PLGA-Ch-GNPs were lyophilized and sealed in sterile glass vials. The vials are then placed in three different storage conditions. The samples were withdrawn at the time intervals 0, 2, 4 and 6 mo and analyzed for PS, \% EE and ZP [32].

\section{In vitro drug release study}

Modified dialysis bag technique was used to determine the in vitro drug release profile of DSB from the optimized DSB-PLGA-Ch-GNPs [33]. DSB-PLGA-Ch-GNPs dispersion was transferred into a dialysis bag (molecular weight cut off between 12-14 kDa) and dialyzed against pH 7.4 PBS $(100 \mathrm{ml})$ on a magnetic stirrer at $37 \pm 0.5^{\circ} \mathrm{C}$ with $100 \mathrm{rpm}$ of stirring. At fixed intervals of time, the whole volume of receptor solutions was replaced with exactly the same volume of fresh pH 7.4 PBS in order to maintain the sink conditions and the solution containing released DSB was quantitatively analyzed by UVVisible spectrophotometer at $325 \mathrm{~nm} \lambda_{\max }[33,34]$. To evaluate the mechanism of DSB release, the in vitro drug release data of the optimized DSB-PLGA-Ch-GNPs was fitted to mathematical models such as the Korsmeyer-Peppas model, Hixon-Crowell cube root model, Higuchi square root model, first-order release model, and zero-order release model [35].

\section{In vitro hemocompatibility study}

In vitro hemocompatibility study was carried out to evaluate the hemolysis. It is necessary for any dosage form that is intended to administer intravenously to exhibit hemocompatibility. Through retro-orbital plexus of rat, the blood sample was collected from and centrifuged at $4000 \mathrm{rpm}$ for $10 \mathrm{~min}$. To the separated erythrocyte sample, the same amount of PBS was added and centrifuged again for $10 \mathrm{~min}$ at $4000 \mathrm{rpm}$. Different concentrations of optimized DSBPLGA-Ch-GNPs $(10-200 \mu \mathrm{g} / \mathrm{ml})$ were added to erythrocyte suspension $(2 \mathrm{ml})$ and incubated at $37{ }^{\circ} \mathrm{C}$ for $45 \mathrm{~min}$ [36]. The resulting samples were stained with hematoxylin and eosin $(\mathrm{H}$ and E) stain, dried on the glass slide and enclosed with a coverslip. Dewinter optical microscope was used to visualize the resulting blood smear samples.

\section{Cellular uptake study by confocal fluorescence microscopy}

The most important and crucial feature that plays a vital role in the success of targeted drug delivery systems is the internalization of the developed nanoparticles. The success of this feature can demonstrate their therapeutic efficacy through intracellular release [37]. K562 human myeloid leukaemia cell lines were cultured, seeded and incubated in multi-well culture plates. The cell line suspension was treated with optimized DSB-PLGA-Ch-GNPs. Meanwhile, the K562 cell line (ACTREC, Mumbai, India) suspension with DSB was taken, and both were incubated at $37{ }^{\circ} \mathrm{C}$ in a $5 \% \mathrm{CO}_{2}$ incubator for $4 \mathrm{~h}$. K562 cells were covered with coverslips, fixed with an aqueous solution of paraformaldehyde (4\% v/v) and washed thrice with PBS solution [38].

\section{In vitro cytotoxicity assay}

It was performed by SRB assay [39]. To determine the cell density on the basis of cell protein content measurement and thereby investigating the cell-based cytotoxicity [40], human myeloid leukaemia cell lines (K562) were maintained in 96 well plates containing RPMI (Roswell Park Memorial Institute)-1640 medium and incubated for $24 \mathrm{~h}\left(37^{\circ} \mathrm{C}, 100 \% \mathrm{RH}, 95 \%\right.$ air and $\left.5 \% \mathrm{CO}_{2}\right)$. After the stipulated time, the well plates containing cells were 
treated with 10, 20, 40 and $80 \mu \mathrm{g} / \mathrm{ml}$ concentrations of experimental drugs (DSB (pure drug), Adriamycin ${ }^{\circledR}$ (standard) and optimized DSB-PLGA-Ch-GNPs) and incubated for $48 \mathrm{~h}$. Cold trichloroacetic acid was mixed to each of the well plates and incubated for 1 hour at $4{ }^{\circ} \mathrm{C}$ in order to fix them in situ. Finally, to stain the cells SRB solution was mixed to the well plates incubated for $20 \mathrm{~min}$ at room temperature. Following staining the cells, the unbound stain was removed, and the bound stain was then eluted with the help of trizma base and the absorbance of the resultant solution was recorded at $540 \mathrm{~nm}$ wavelength. Percentage cell growth and percentage growth inhibition were calculated by using the formula:

$$
\begin{gathered}
\% \text { Cell growth }=\frac{\text { average absorbance of the test wells }}{\text { average absorbance of the control wells }} \times 100 \\
\% \text { Growth inhibition }=100-\% \text { cell growth }
\end{gathered}
$$

\section{Cell apoptosis assay}

Apoptosis or programmed cell death is the process of removal of unwanted cells, thereby conserving the key proteins. Apoptosis occurs due to a cascade of events, and apoptotic cells can be identified by morphological features like cell shrinkage, chromatin condensation, nuclear envelope disintegration and formation of irregular bulges in the cell membrane. Flow cytometry was employed to quantify the total number of apoptotic cells in a clinical sample by making measurements on each individual cell [41]. To investigate the mode of cell death, K562 cells were cultured and at 1 x $10^{5}$ cells/well density, the cells were carefully seeded into the multi-well plates containing 10\% fetal bovine serum (FBS) in RPMI medium. K562 cells were treated with $0,5,10$ and $15 \mu \mathrm{g} / \mathrm{ml}$ concentrations of optimized DSB-PLGA-Ch-GNPs and incubated for 6,12 and $24 \mathrm{~h}$ in an incubator contained with $5 \% \mathrm{CO}_{2}$ at $37^{\circ} \mathrm{C}$. Then K562 cells were collected, thoroughly washed with ice-cold PBS and re-suspended in $1 \mu \mathrm{g} / \mathrm{ml}$ concentration of propidium iodide and annexin V-fluorescein isothiocyanate binding buffer. After that, cell specimens were subjected to incubation in a light-protected area at room temperature for 15 min and analyzed by using BD FACS Calibur ${ }^{\mathrm{TM}}$ flow cytometry system.

\section{RESULTS AND DISCUSSION}

\section{Formulation of DSB-PLGA-Ch-GNPs}

The synthesized Ch-GNPs were visually observed for any color change in the dispersion. As a result of bioreduction by chitosan, a ruby red color was produced within 1 hour. It indicated the green synthesis of Ch-GNPs, which was confirmed by examining the resultant dispersion UV-Visible spectrophotometry in the scanning range of $300-800 \mathrm{~nm}$. A peak maximum was recorded at $525 \mathrm{~nm}$ (SPR band of GNPs) in the UV-Visible absorption spectrum. Subsequently, stabilization and loading of DSB were done after the successful synthesis of Ch-GNPs and DSB was selected as an anticancer drug for loading GNPs to achieve targeted drug delivery.

\section{Risk assessment studies}

The identification of CQAs that might influence the quality of the nanoformulation is a prerequisite of $\mathrm{QbD}$. The essential CQAs were prioritized by FMEA based on rank modes of relative effectiveness as low, medium and high. The results obtained by employing DOE to optimize the GNPs manufacturing process were further subjected to design screening.

\section{Risk assessment screening}

As the number of independent variables was more, risk assessment screening was performed to decrease the sets of independent variables to those that have the most influence on the dependent variables. Using Minitab 17 software screening was done by 8 factors, 2 level PBD. Polymer concentration (PC), stirring time (ST), stirring speed (SS), sonication frequency (SF), sonication time (SoT), temperature (T), centrifugation speed (CS), and centrifugation time (CT), are the independent variables used in the experimentation. All the unique properties of the GNPs are influenced by its particle size. PC, ST and SoT were identified as the most significant variables through preliminary screening by PBD.

The rate and extent of uptake of the drug in the cellular microenvironment is the main aspect that decides the therapeutic efficacy of the DSB-PLGA-Ch-GNPs. In order to attain this, nanoparticles with appropriate \% EE and good release from the nanocarrier, ensuring the sustained concentration of DSB at the site of uptake is desirable. The long-term physical stability of the prepared nanoformulation is necessary, and zeta potential is a key indicator for ensuring it. Hence, PS, \% EE and ZP were selected as responses (dependent variables). 12 PBD experimental runs were generated with PS, \% EE and ZP. The results of observed mean values of PS was found to be in the range of $12.18 \pm 0.52$ to $32.98 \pm 0.32 \mathrm{~nm}, \%$ EE in the range of $42.24 \pm 0.35$ to $79.84 \pm 0.42 \%$ and ZP in the range of $-10.56 \pm 0.08$ to- $18.14 \pm 0.02 \mathrm{mV}$. ANOVA was employed to evaluate the significance of PBD and statistically significant coefficients for each factor. Subsequently, Pareto charts were generated in order to find the main significant factors influencing the responses (fig. 1).

\section{$2^{3}$ FFD}

The formulation design was carried out using the response surface method by 3 factor, 2 level, and 3 response full factorial design and systematically explored the main, quadratic and interaction effects of each individual factor on the responses. A total of 8 randomized experimental runs were produced, and the experiments were performed accordingly. Experimental runs were conducted, and the results of PS, \% EE and ZP are shown in table 2. The regression equations were generated for each response, and a ' + ' symbol in front specifies synergistic effect while ' - ' symbol specifies the antagonistic effect of the independent variables [42]. 3D response surface plots were used to enumerate the interaction and correlation among the factors and the measured responses. ANOVA was used to carry out the data analysis and estimation of the quantitative effect of the factors.

\begin{tabular}{|c|c|c|c|c|c|c|}
\hline Run & PC & ST & SoT & PS* & \% EE* & ZP* \\
\hline FFD-1 & 0.10 & 8 & 10 & $29.48 \pm 0.94$ & $76.84 \pm 0.27$ & $-12.97 \pm 1.04$ \\
\hline FFD-2 & 0.01 & 4 & 30 & $26.36 \pm 1.24$ & $65.87 \pm 0.92$ & $-16.37 \pm 1.08$ \\
\hline FFD-3 & 0.10 & 4 & 10 & $31.84 \pm 1.04$ & $73.54 \pm 0.28$ & $-10.37 \pm 1.08$ \\
\hline FFD-4 & 0.01 & 8 & 30 & $24.18 \pm 1.52$ & $69.28 \pm 0.94$ & $-18.54 \pm 1.06$ \\
\hline FFD-5 & 0.10 & 8 & 30 & $28.96 \pm 1.28$ & $78.62 \pm 0.48$ & $-13.28 \pm 1.02$ \\
\hline FFD-6 & 0.10 & 4 & 30 & $30.82 \pm 1.32$ & $74.31 \pm 0.64$ & $-11.08 \pm 1.12$ \\
\hline FFD-7 & 0.01 & 4 & 10 & $26.98 \pm 1.18$ & $62.98 \pm 0.76$ & $-15.96 \pm 1.04$ \\
\hline FFD-8 & 0.01 & 8 & 10 & $25.22 \pm 1.62$ & $68.48 \pm 0.94$ & $-17.26 \pm 1.06$ \\
\hline
\end{tabular}

Table 2: $2^{3}$ full factorial design matrix and results of observed mean values of various responses

$\left({ }^{*}\right.$ mean $\pm \mathrm{SD}, \mathrm{n}=3$ ) [PC (polymer concentration), ST (stirring time), SoT (sonication time), PS (particle size), \% EE (\% entrapment efficiency), and ZP (zeta potential)]. 

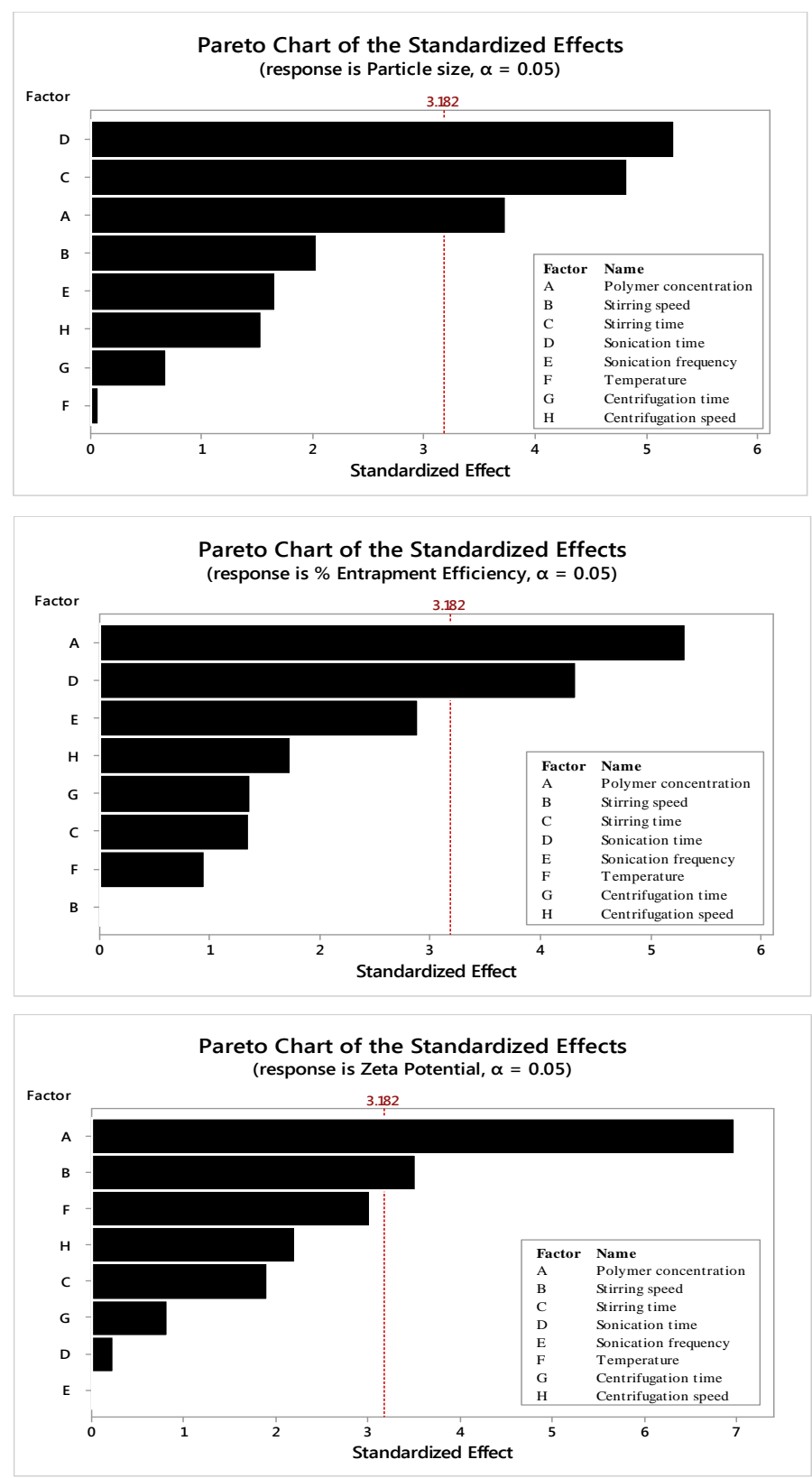

Fig. 1: Pareto charts showing the influence of significant independent variables on dependent variables

PS is a significant CQA which influences the drug payload, biodistribution, ability to target and toxicity. According to the $2^{3} \mathrm{FFD}$, the mean PS of the developed DSB-PLGA-Ch-GNPs ranged between $31.84 \pm 1.04 \mathrm{~nm}$ to $24.18 \pm 1.52 \mathrm{~nm}$ (table 2). The following regression equation illustrates the effect of independent variables on the PS:

\section{PS $=28.16+68.00$ PC-0.358 ST-0.0026 SoT-2.944 PC. ST-0.733 PC. SoT- 0.0065 ST. SoT+0.127 PC. ST. SoT}

It was found that there was a significant effect of PC on the PS. A synergistic effect was noticed between PC and PS, such that an increase in PC resulted in an increase in PS. As reported by previous researchers, an increase in the PC has a synergistic effect on PS [43]. Similarly, Vardhan et al. also observed that at a higher PC, the viscosity of organic phase increases, which resists the development of the nanoparticulate system to break, resulting in larger nanoparticles [28]. The relationship between the factors and PS was further explained by 3D response surface plots, and its corresponding graphs are shown in fig. 2 (A and B). Although, the PS increased at increased PC; however, the final size of the nanoparticles was affected by the integrated effect of the ST as well as the SoT. As a matter of fact, more ST leads to high mechanical and hydraulic shear and can impressively decrease the PS.
According to the $2^{3} \mathrm{FFD}$, the $\%$ EE of the developed DSB-PLGA-ChGNPs ranged between $78.62 \pm 0.48 \%$ to $62.98 \pm 0.76 \%$ (table 2 ). The impact of PC on the \% EE was more significant than the ST and SoT, as an increase in PC caused an increase in PS and subsequently led to increased \% EE. Furthermore, the use of high molecular weight polymer in the nanoformulation resulted in improved \% EE and this outcome was similar to that observed by Viswanadh et al. [44]. The following regression equation illustrates the effect of independent variables on the $\% \mathrm{EE}$ :

\section{$\% \mathrm{EE}=53.28+170.8 \mathrm{PC}+1.740 \mathrm{ST}+0.278$ SoT-10.42 PC. ST-2.90 PC. SoT-0.030 ST. SoT+0.430 PC. ST. SoT}

The \% EE was decreased at a high ST, which was primarily because of the diffusion of the DSB out of the nanoparticles during the size reduction stage. 3D response surface plot of the DSB-PLGA-Ch-GNPs is shown in fig. 2 (C). The results indicated that at high PC, ST and SoT the \% EE showed maximum values and at high ST and low SoT, the $\% \mathrm{EE}$ was decreased.

According to the $2^{3} \mathrm{FFD}$, the ZP of the developed DSB-PLGA-Ch-GNPs was in the range of-18.54 $\pm 1.06 \mathrm{mV}$ T0-10.37 $\pm 1.08 \mathrm{mV}$ (table 2 ). The 
following regression equation illustrates the effect of independent variables on the ZP:

\section{$\mathrm{ZP}=15.74-85.28 \mathrm{PC}+0.162 \mathrm{ST}-0.0317 \mathrm{SoT}+5.375 \mathrm{PC} . \mathrm{ST}+0.872 \mathrm{PC}$ SoT+0.0126 ST. SoT-0.176 PC. ST. SoT}

3D response surface plot of the DSB-PLGA-Ch-GNPs is shown in fig. 2 (D). The quadratic model's ANOVA analysis generated for PS, \% EE and ZP indicated an excellent fit between predicted values and experimental values, there was no lack of fit $(>0.05)$.

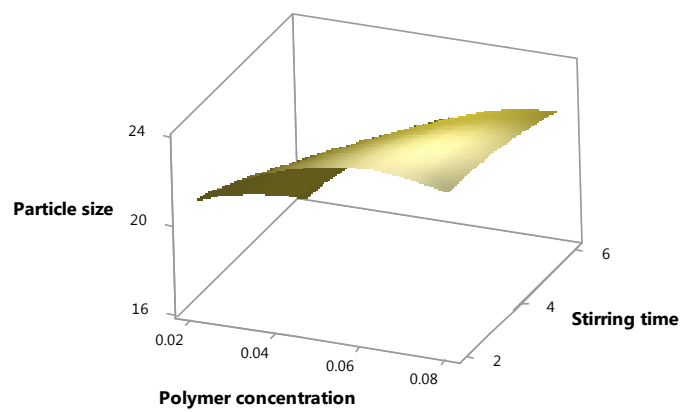

(A)

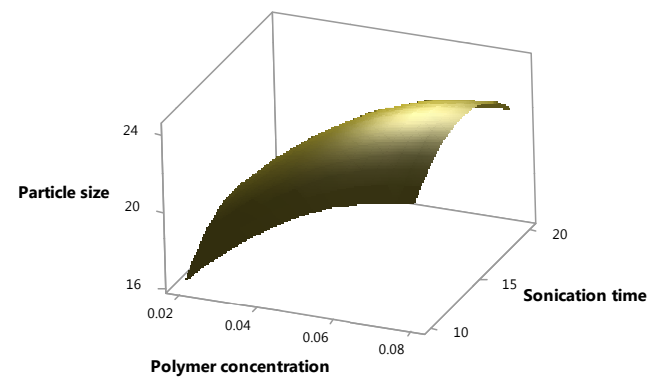

(B)

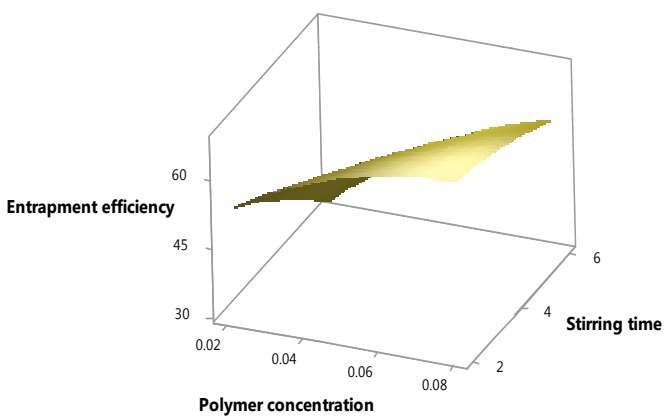

(C)

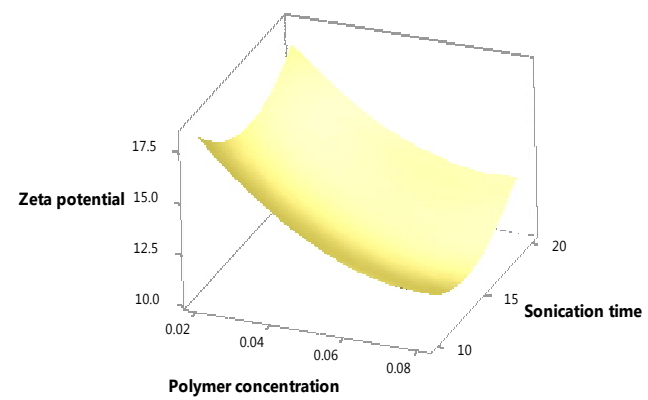

(D)

Fig. 2: The 3D response surface plots showing the effect of polymer concentration, stirring time and sonication time on particle size (A and B), \% entrapment efficiency (C) and zeta potential (D)
Response optimization was carried out in order to get a better nanoformulation with suitable PS, \% EE and ZP. $0.098 \% \mathrm{w} / \mathrm{w}, 8 \mathrm{~h}$ and 30 min were defined as the optimum points for the PC, ST and SoT respectively. PS, \% EE and ZP were predicted as. $28.88 \mathrm{~nm}$, $78.47 \%$ and $-13.36 \mathrm{mV}$, respectively.

Physicochemical and morphological characterization of optimized DSB-PLGA-Ch-GNPs

\section{$P S, \% E E$ and ZP}

The mean PS of the optimized DSB-PLGA-Ch-GNPs was found to be $26.94 \pm 1.14 \mathrm{~nm}$. The PS of nanoformulation plays a pivotal role in targeted drug delivery. The mean \% EE of the optimized DSB-PLGACh-GNPs was found to be $80.72 \pm 0.58 \%$. High \% EE is desirable as it carries a high drug payload and influences the rate and extent of uptake of the drug in the cellular microenvironment, the therapeutic efficacy of the nanoformulation and ultimately the sustained release of drug at the site of uptake. The mean ZP of the optimized DSBPLGA-Ch-GNPs was found to be- $15.46 \pm 1.06 \mathrm{mV}$. ZP of the optimized DSB-PLGA-Ch-GNPs was within the acceptable limits, and it is a key indicator for ensuring the long-term physical stability of the prepared nanoformulation.

\section{FTIR}

FTIR overlay spectrum of the optimized DSB-PLGA-Ch-GNPs along with DSB, PLGA, Ch, and GNPs is shown in fig. 3 . The characteristic bands in the FTIR spectrum of DSB were found at $1620.26 \mathrm{~cm}^{-1}$ (C 0 stretching), $2949.26 \mathrm{~cm}^{-1}$ (C-H stretching), $3026.41 \mathrm{~cm}^{-1}(-\mathrm{C}-\mathrm{H}$ aromatic ring), $3201.94 \mathrm{~cm}^{-1}$ (O-H stretching), and $3417.98 \mathrm{~cm}^{-1}$ (N-H stretching). For chitosan spectrum, the bands were observed at $1031.95 \mathrm{~cm}^{-1}$ (C-O stretching), $1321.28 \mathrm{~cm}^{-1}$ (C-H), 1564.32 $\mathrm{cm}^{-1}(\mathrm{~N}-\mathrm{H}), 1654.98 \mathrm{~cm}^{-1}(-\mathrm{NH} 2)$, and $3385.18 \mathrm{~cm}^{-1}(-\mathrm{N}-\mathrm{H})$. In the case of PLGA the bands appeared at $750.33 \mathrm{~cm}^{-1}$ ( $\mathrm{C}-\mathrm{H}$ bending), $1047.38 \mathrm{~cm}^{-1}$ (-C-CH3), $1186.26 \mathrm{~cm}^{-1}$ (-C-O stretching), 1384.94 $\mathrm{cm}^{-1}$ (O-H bending), $1456.30 \mathrm{~cm}^{-1}$ (C-H stretching), and 1755.28 $\mathrm{cm}^{-1}(-\mathrm{C}=0$ stretching). All the characteristic bands of DSB were retained in the optimized DSB-PLGA-Ch-GNPs FTIR spectrum. Further, it suggested that DSB did not interact with other components, and there was no change in its chemical nature during the formulation development.

\section{XRD}

XRD overlay spectrum of optimized DSB-PLGA-Ch-GNPs along with DSB, PLGA, Ch, and GNPs is shown in fig. 4. XRD spectrum of DSB exhibited its crystalline nature with well resolved and relatively intense peaks at $2 \theta$ angles of $21.11^{\circ}, 21.85^{\circ}, 22.11^{\circ}, 23.13^{\circ}, 24.42^{\circ}$, $25.32^{\circ}, 27.81^{\circ}$. PLGA, Ch, and Ch-GNPs diffraction patterns displayed numerous small diffuse peaks with a broad halo. DSB-PLGA-Ch-GNPs spectrum exhibited broad diffuse peaks which suggest that the crystalline nature of the DSB was decreased and converted into amorphous nature.

\section{EDXS}

Successful reduction of the GNPs precursor was confirmed by sharp and clear gold $(\mathrm{Au})$ peaks in the EDXS spectrum (fig. 5 (B)). Besides, copper $(\mathrm{Cu})$ and carbon $(\mathrm{C})$ peaks were also present in the EDXS spectrum due to the use of the carbon-coated copper grid on to which the optimized DSB-PLGA-Ch-GNPs was placed. Tahir et al. also observed the similar strong signals for gold atoms in their EDXS spectrum [45].

\section{TEM and SAED}

TEM micrographs revealed that the grains of the optimized DSBPLGA-Ch-GNPs were nanosized, spherical, smooth-surfaced and uniformly distributed. A clear core-shell structure with a lighter outer envelop and a dark inner core was found because of the electron density differences in outer envelop and the gold core (fig. 6 (A)). Pertiwi et al. also observed spherical and mostly separated gold nanoparticles when they biosynthesized gold nanoparticles by green reduction method using an aqua extract [46]. Further, the optimized DSB-PLGA-Ch-GNPs showed bright circular spots and characteristic diffraction ring patterns in the SAED confirming the presence of gold and amorphous material, respectively (fig. 6 (B)). Devi et al. 
developed gemcitabine hydrochloride-loaded colloidal gold nanoparticles. The SAED pattern of their formulation showed diffraction spots, which indicated an electrostatic interaction between the drug and gold nanoparticles and confirms the drug presence on gold nanoparticles. Here also, similar diffraction spots were observed, which confirms the presence of DSB on GNPs [47].
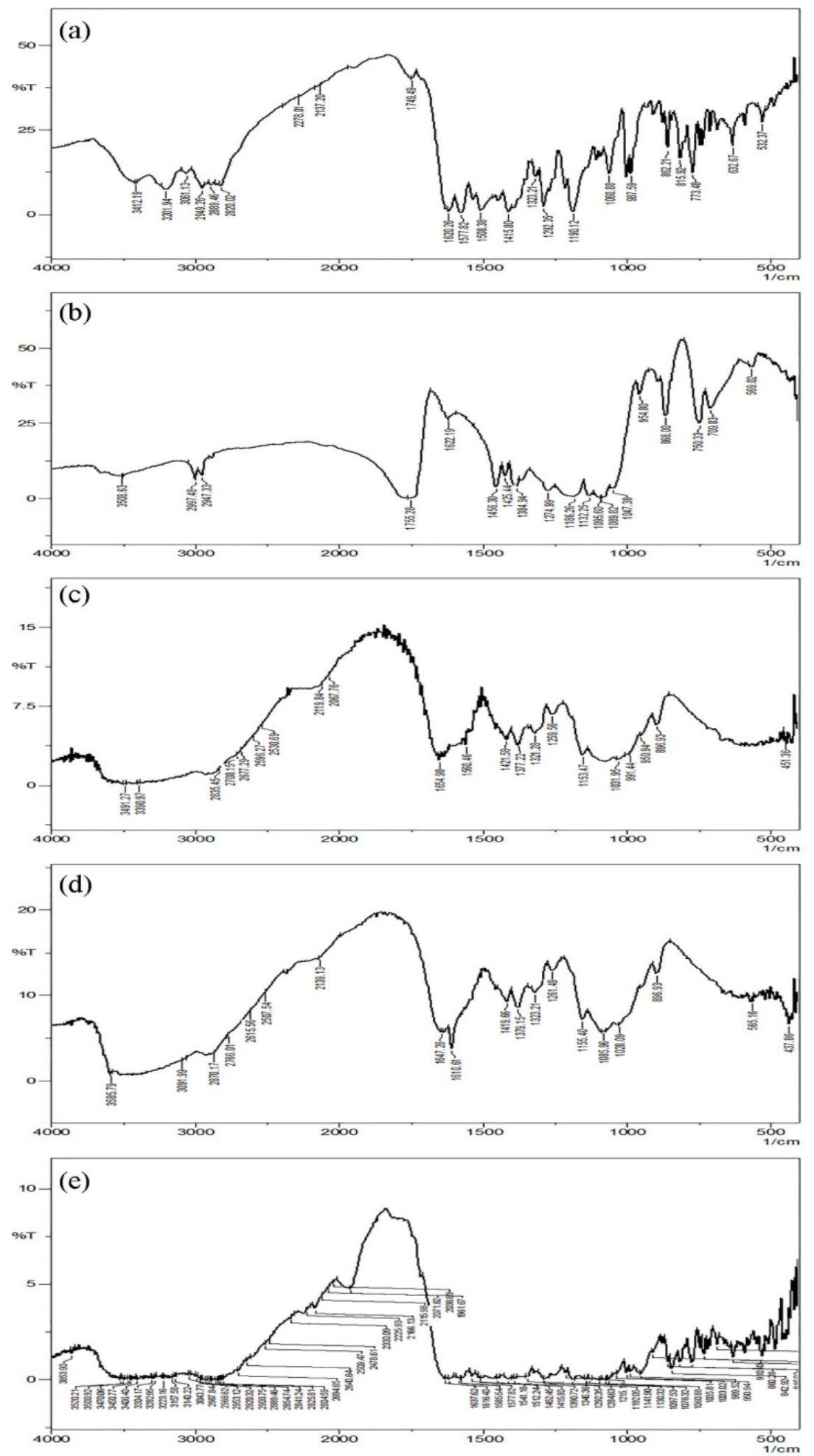

Fig. 3: FTIR overlay spectrum of DSB-PLGA-Ch-GNPs, (a) DSB; (b) PLGA; (c) Ch; (d) GNPs and (e) Optimized nanoformulation

\section{AFM}

3D and 2D surface topographic AFM micrographs of the optimized DSB-PLGA-Ch-GNPs showed uniform, smooth-surfaced spherical nanoparticles without any perforations and agglomerates (fig. 7 (A and B)). This was achieved mainly due to the stabilization and uniform covering by the PLGA over the nanoparticle's surface. Rao et al. also observed such smooth spherical surface morphologies for their gum tragacanth stabilized Naringin-loaded gold nanoparticles [48]. 

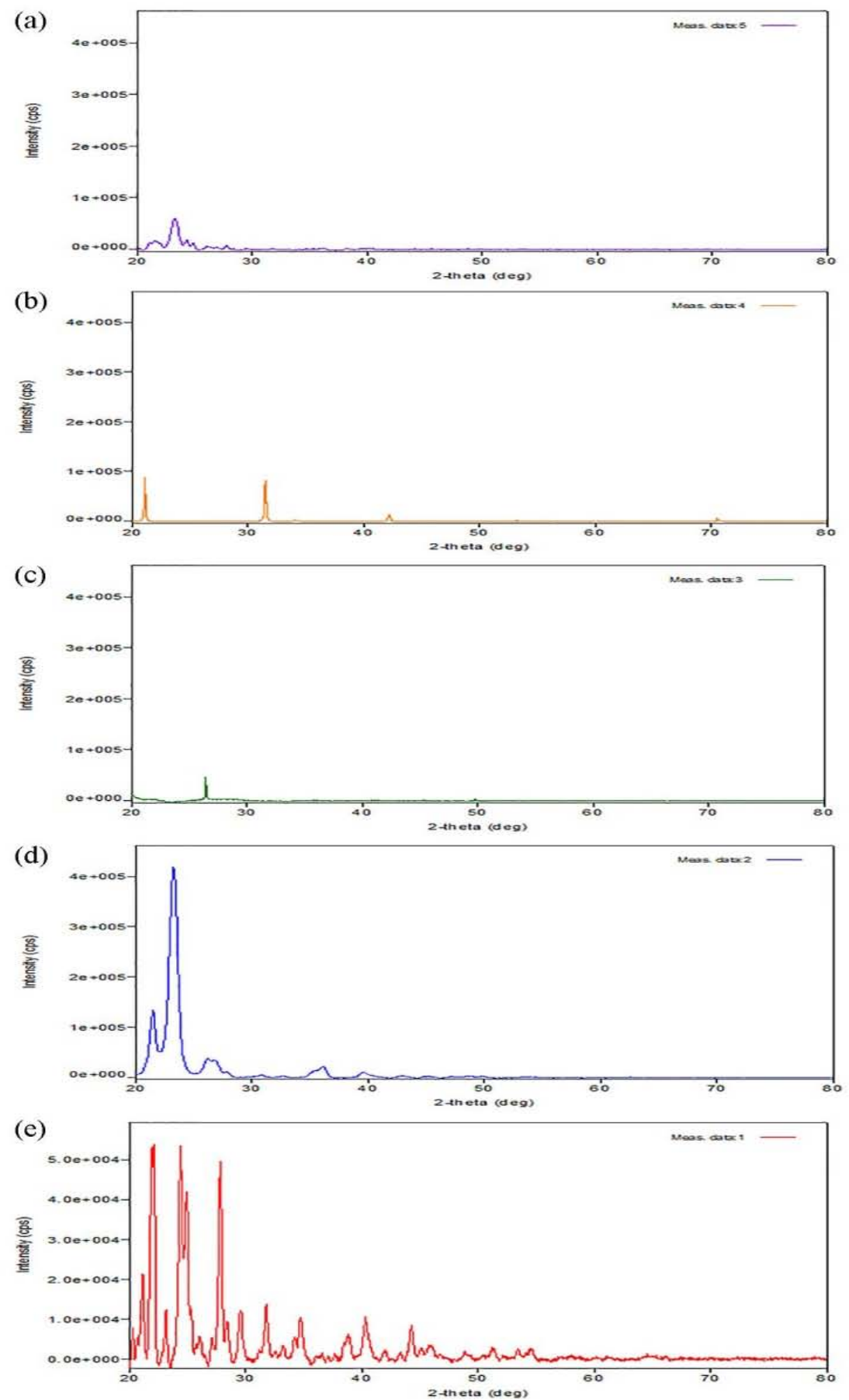

Fig. 4: XRD overlay spectrum of DSB-PLGA-Ch-GNPs, (a) DSB; (b) PLGA; (c) Ch; (d) GNPs and (e) Optimized nanoformulation

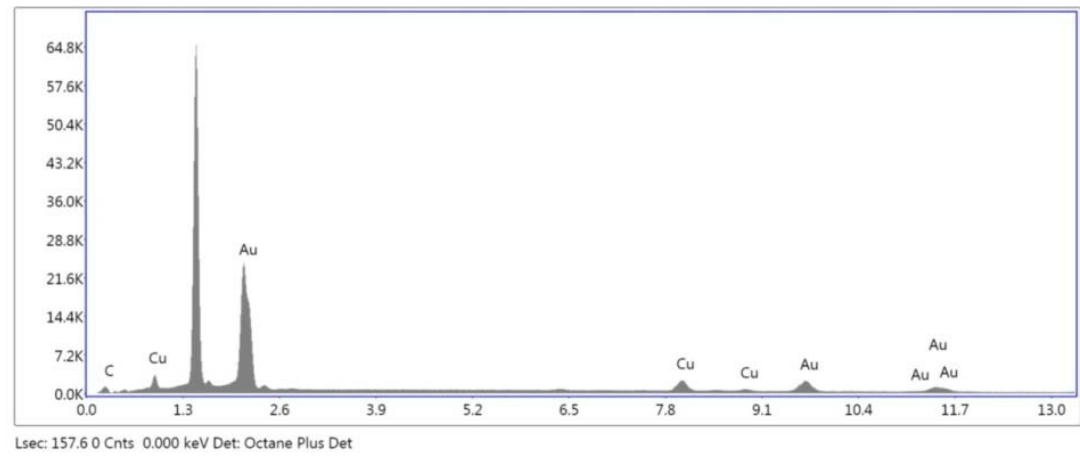

Fig. 5: EDXS spectrum of the optimized DSB-PLGA-Ch-GNPs 


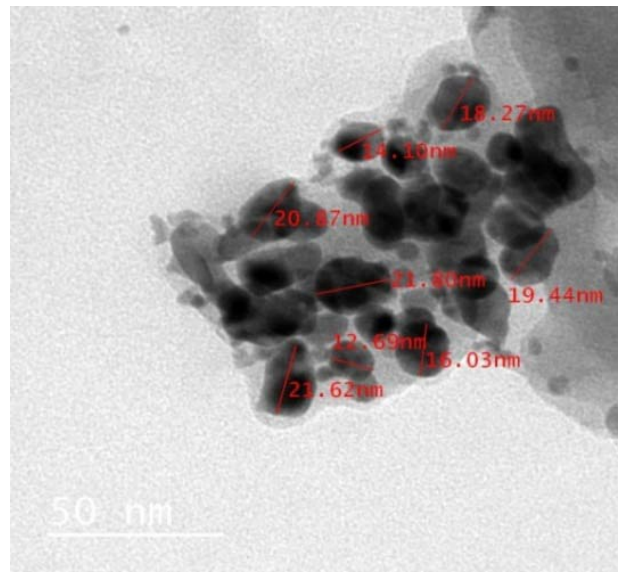

(A)

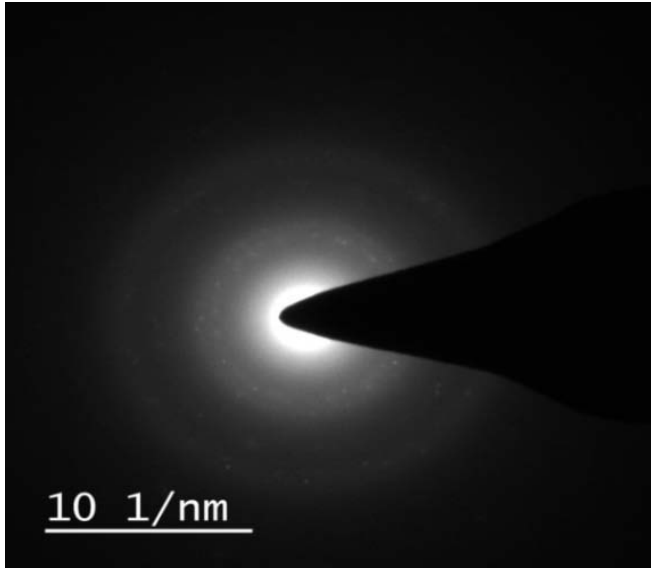

(B)

Fig. 6: TEM micrograph (A) and SAED (B) of the optimized DSB-PLGA-Ch-GNPs

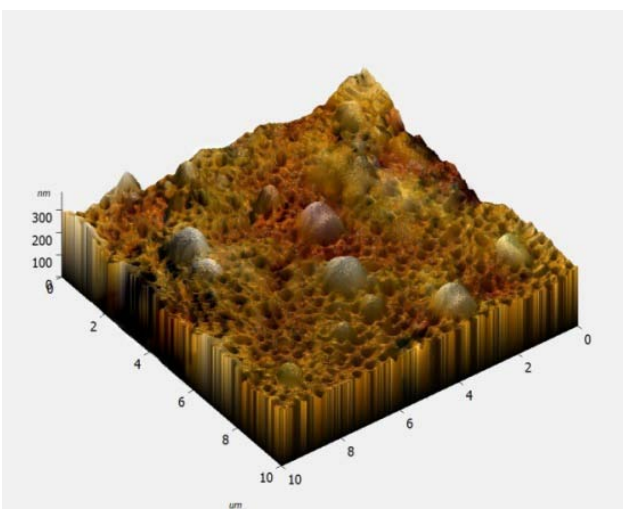

(A)

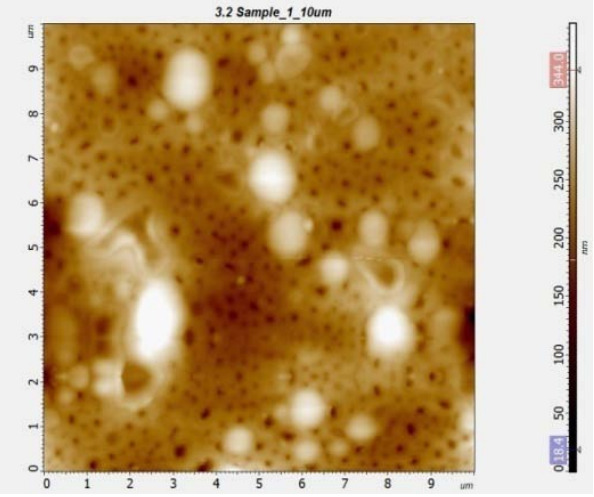

(B)

Fig. 7: 3D AFM micrograph (A) and 2D AFM micrograph (B) of the optimized DSB-PLGA-Ch-GNPs

\section{Stability study}

The optimized DSB-PLGA-Ch-GNPs were subjected to stability testing at $40{ }^{\circ} \mathrm{C} \pm 2{ }^{\circ} \mathrm{C} / 75 \% \mathrm{RH} \pm 5 \% \mathrm{RH}$ (accelerated), $25{ }^{\circ} \mathrm{C} \pm 2{ }^{\circ} \mathrm{C}$ (room temperature) and $4{ }^{\circ} \mathrm{C} \pm 1{ }^{\circ} \mathrm{C}$ (refrigerated) conditions for a period of $6 \mathrm{mo}$ and the results are shown in fig. 8. No significant change in the physical appearance as well as in the PS, \% EE and ZP of the optimized DSB-PLGA-Ch-GNPs was observed at the accelerated condition, room temperature and refrigerated condition over a period of $6 \mathrm{mo}$. Vardhan et al. carried out stability studies to determine the effect of stress conditions on the nanoformulations under different storage conditions and the results showed that there was no significant change in the PS, \% EE and ZP [28].

\section{In vitro drug release study}

The results of the in vitro drug release study of the optimized DSBPLGA-Ch-GNPs (fig. 9) showed $32.06 \%$ of the drug release in the first $4 \mathrm{~h}$, and $79.34 \%$ of the drug release by the end of $48 \mathrm{~h}$. Thus, it was inferred that the drug release from the optimized DSB-PLGA-ChGNPs exhibited a sustained-release pattern. It was achieved mainly because of a strong drug-polymer interaction. Niza et al. also observed similar kind of sustained drug release profile from dasatinib loaded trastuzumab conjugated nanoparticles [49]. The mechanism of DSB release from the optimized DSB-PLGA-Ch-GNPs was best elucidated by Korsmeyer-Peppas model $\left(R^{2}=0.978\right)$.

\section{In vitro hemocompatibility study}

For any dosage form that is intended to administer intravenously, the evaluation of hemolysis is mandatory. An in vitro hemocompatibility study was carried out for the optimized DSBPLGA-Ch-GNPs to test the hemolytic potential. The results showed that there was no sign of hemolysis when different concentrations of optimized DSB-PLGA-Ch-GNPs were added to the erythrocyte suspension. This indicates that the optimized DSB-PLGA-Ch-GNPs exhibit no hemolysis after intravenous administration and makes a safe, compatible, and suitable candidate for the application. Chen et al. also observed negligible hemolysis $(<1 \%)$ after treatment with dasatinib-loaded nanoparticles at high concentrations, demonstrating excellent hemocompatibility and the nanoformulation is suitable for i. v. administration [50].

\section{Cellular uptake study by confocal fluorescence microscopy}

K562 human myeloid leukaemia cell lines treated with the drug and optimized DSB-PLGA-Ch-GNPs were used for the cellular uptake study. K562 cells after incubation with DSB and optimized DSBPLGA-Ch-GNPs are shown in fig. 10. The fluorescence of the GNPs was higher and showed more intensity than that of the DSB. It was observed that the optimized DSB-PLGA-Ch-GNPs showed approximately 10 to 20 fold much higher intracellular fluorescence intensities than that of DSB (pure drug) alone for the most part of the K562 cell line. These results indicated that through GNPs the DSB from the DSB-PLGA-Ch-GNPs can readily permeate and accumulate into the leukaemia cancer cells and subsequently act as an effective nanocarrier system to assist the targeted DSB delivery. Mandal et al. also observed percentage of cells that internalized the functionalized GNPs through confocal microscopy and the results confirmed enhanced uptake of GNPs by different cancer cell lines and assist in targeted delivery [51]. 

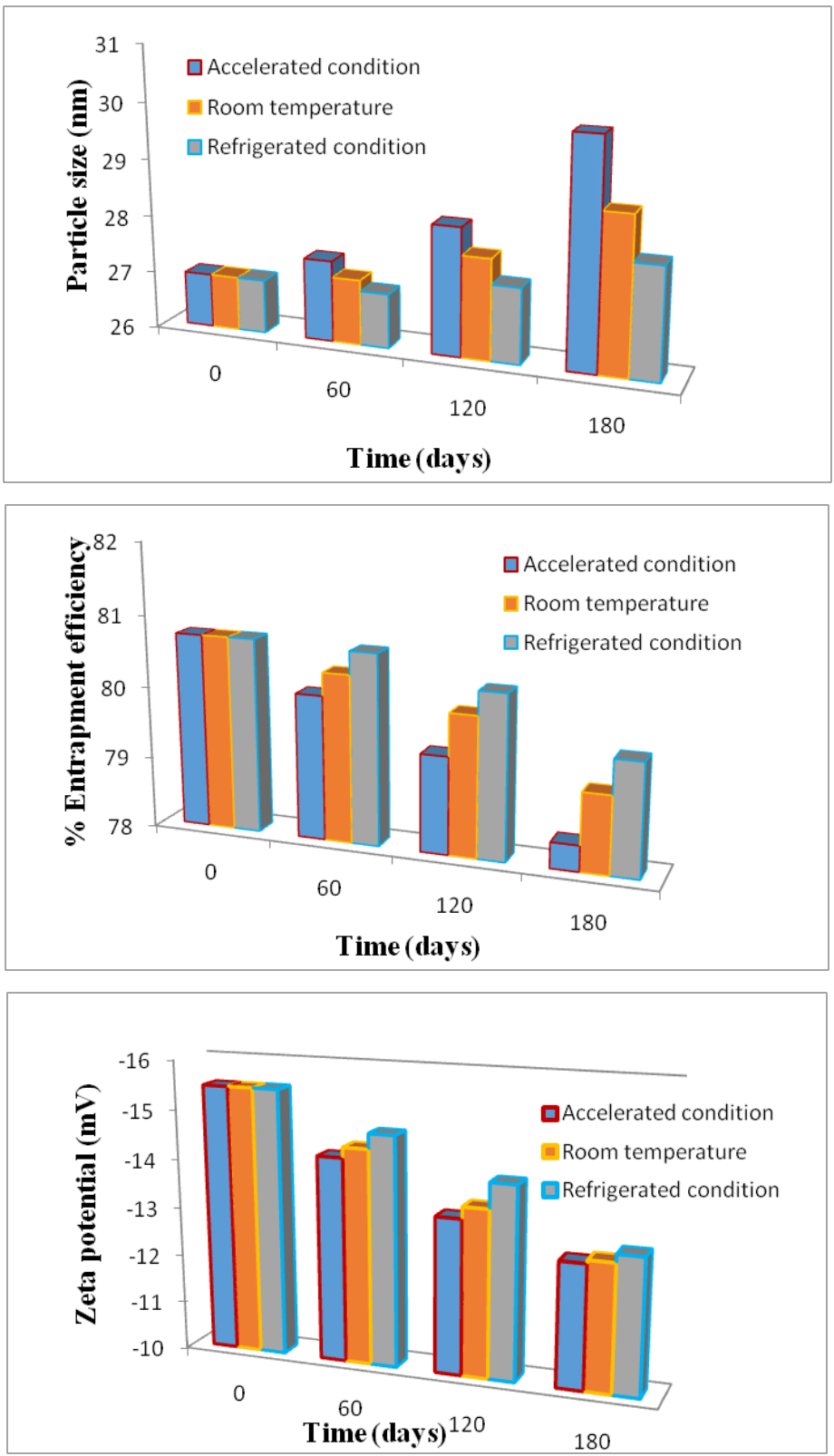

Fig. 8: Stability study profiles of the optimized DSB-PLGA-Ch-GNPs, (vertical bars represents mean \pm SD and $\mathbf{n}=3$ )

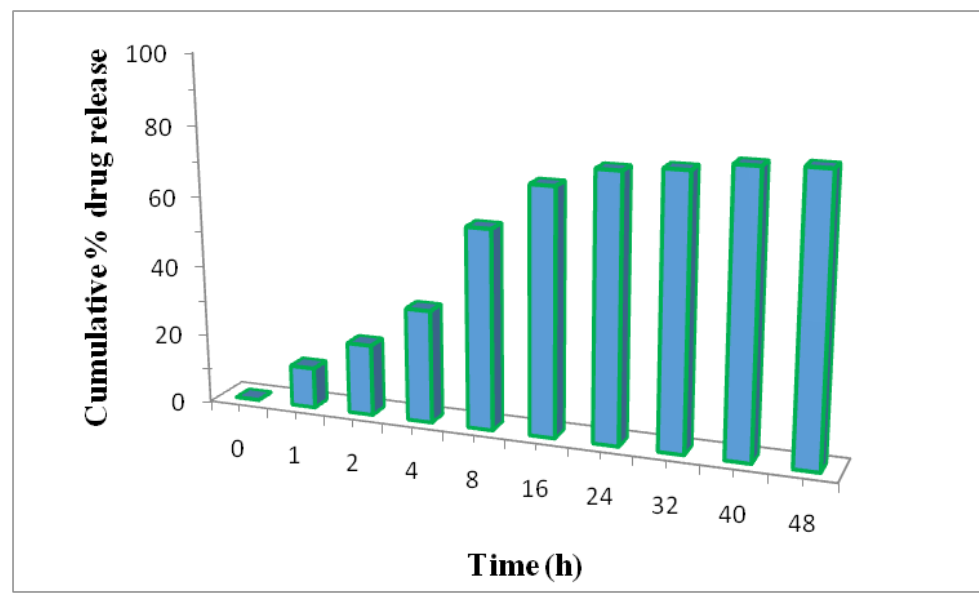

Fig. 9: In vitro drug release profile of the optimized DSB-PLGA-Ch-GNPs in pH 7.4 PBS, (vertical bars represents mean \pm SD and $n=3$ ) 


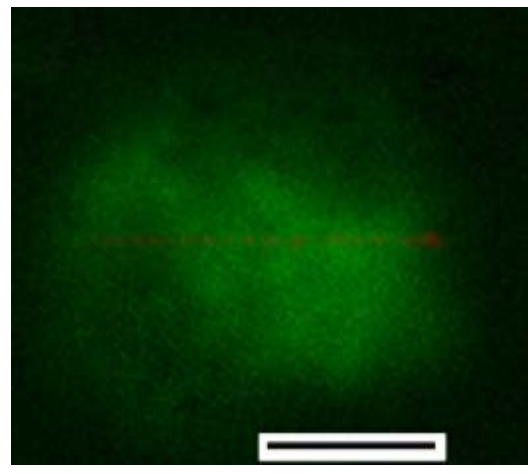

(A)

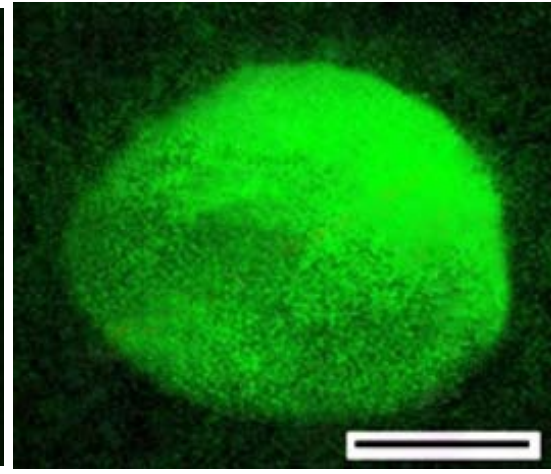

(B)

Fig. 10: Confocal microscopic images of DSB (A) and the optimized DSB-PLGA-Ch-GNPs (B) in K562 cell line

\section{In vitro cytotoxicity assay}

To examine the cell viability in vitro cytotoxicity study was performed for the optimized DSB-PLGA-Ch-GNPs, reference standard and pure drug by SRB assay. It was performed against K562 human myeloid leukaemia cell lines to compare the percentage cell growth and percentage growth inhibition by the optimized DSB-PLGA-Ch-GNPs as compared to control, standard drug (adriamycin) and the pure drug (DSB). The percentage control growth vs drug concentration profiles are shown in fig. 11. The optimized DSB-PLGA-Ch-GNPs exhibited significantly more \% growth inhibition as compared to DSB. This indicates that the optimized DSB-PLGA-Ch-GNPs exhibits a potential cytotoxic effect in the treatment of CML. Thapliyal et al. also observed enhanced \% growth inhibition of nanoformulation as compared to the pure drug in a dose-dependent manner [52].

\section{Cell apoptosis assay}

Cell apoptosis assay is based on a study of a total cell population that averages the results from every given cell. The effect of the optimized DSB-PLGA-Ch-GNPs on the induction of apoptosis was evaluated with PI and annexin V-FITC by increasing concentration of the optimized DSB-PLGA-Ch-GNPs $(0,5,10$ and $15 \mu \mathrm{g} / \mathrm{ml})$ and exposure time $(6,12$ and $24 \mathrm{~h}$ ). The flow cytometry results revealed that with the increase in the optimized DSB-PLGA-Ch-GNPs concentration and exposure time, the cell death was also increased in the cell population, suggesting that the optimized DSB-PLGA-Ch-GNPs could exert effective antileukaemia activity against K562 cells and further induce the K562 cell death with a dose and time-dependent manner (fig. 12 and fig. 13). Debrala et al. also observed that MCF-7 cells treated with formulation brought about an extended mitotic arrest, which is a typical morphological sign of apoptosis [53].

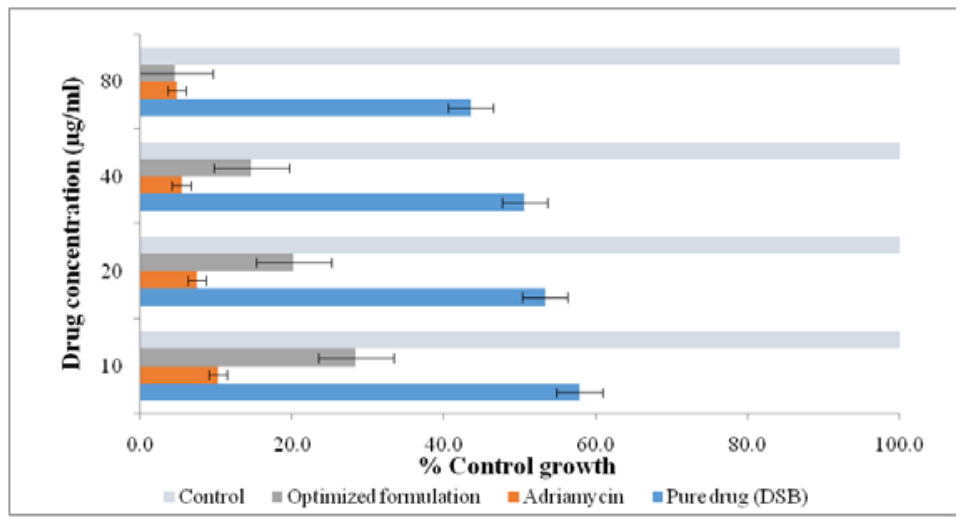

Fig. 11: Percentage control growth versus drug concentration $(\mu \mathrm{g} / \mathrm{ml})$ profiles, (vertical bars represents mean $\pm S D, n=3, p<0.001$ compared to pure drug, Two-way ANOVA, Bonferroni post-test)

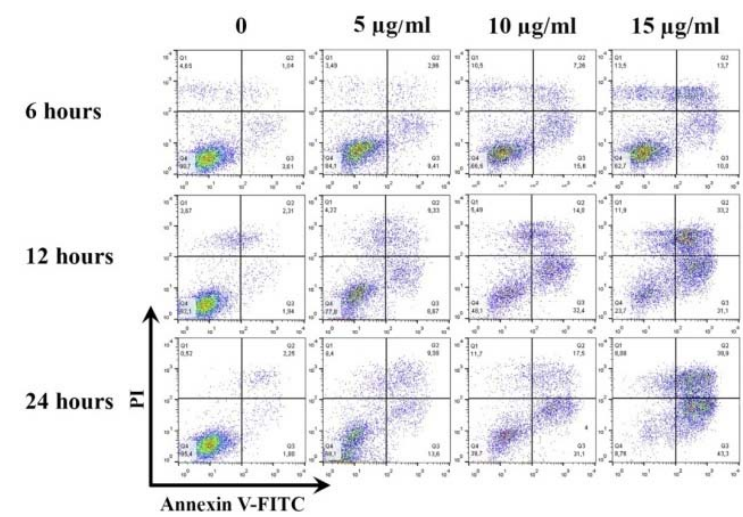

Fig. 12: Dot plot of apoptosis in K562 cell line detected by flow cytometry 


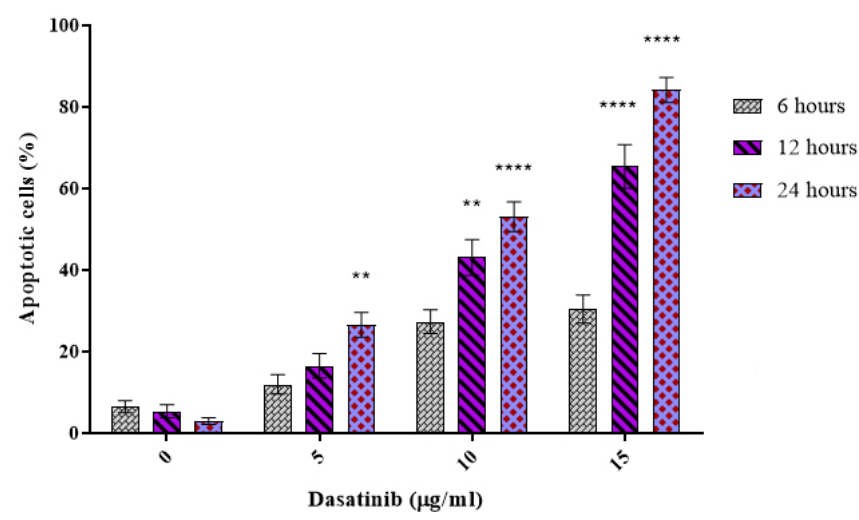

Fig. 13: Bar graph displaying the percentage of apoptotic cells at different concentrations of the optimized DSB-PLGA-Ch-GNPs and exposure times, (vertical bars represents mean $\pm \mathrm{SD}, \mathrm{n}=4,{ }^{* * * *} \mathrm{p}<0.0001, * * \mathrm{p}<0.01$, Bonferroni post-test)

\section{CONCLUSION}

Conclusively, the present research embodies to design, develop, optimize and characterize DSB-PLGA-Ch-GNPs for improved therapy of CML. In this study, targeted drug delivery of DSB loaded polymeric GNPs was attempted to enhance its therapeutic efficiency and reduce the adverse effects. The anticipated range of PS, \% EE and ZP was found in the desired range. The physicochemical and morphological characterization studies demonstrated smooth and spherical shape without any aggregation. The majority of the drug amount had been converted into an amorphous form due to interaction with the polymer. The in vitro drug release profile showed a sustained-release pattern, and the drug release followed the Korsmeyer-Peppas mechanism. The hemocompatibility results showed that the optimized DSB-PLGA-Ch-GNPs is a suitable candidate for the application as there was no sign of hemolysis. In vitro cytotoxicity study indicated that the optimized DSB-PLGA-ChGNPs exhibited a potential cytotoxic effect in the treatment of CML. Cellular uptake study confirmed that the DSB loaded GNPs can act as an effective nanocarrier system to assist the targeted DSB delivery. The cell apoptosis assay revealed that the optimized DSB-PLGA-ChGNPs could provide effective antileukaemia activity against K562 cells and further induce the K562 cell death with dose and timedependent manner. It can be concluded that the prepared DSBPLGA-Ch-GNPs in this research offered significant advantages, including sustained drug release, making it suitable for DSB delivery with improved biocompatibility, stability, and therapeutic efficiency.

\section{ACKNOWLEDGMENT}

The authors acknowledge the help of MSN Laboratories Pvt. Ltd, (Hyderabad, Andhra Pradesh, India) for providing dasatinib drug as a gift sample.

\section{FUNDING}

Nil

\section{AUTHORS CONTRIBUTIONS}

Sandeep Kumar Reddy Adena conceived the idea, conducted all the experiments, wrote the manuscript, reviewed and finalized the manuscript. He is accountable for all aspects of the work in ensuring questions related to the accuracy or integrity of any part of the work are appropriately investigated and resolved. Kasi Viswanadh Matte assisted in preparing the manuscript. Ramoji Kosuru assisted in acquiring and interpreting the data.

\section{CONFLICT OF INTERESTS}

The authors declare that they have no conflicts of interest.

\section{REFERENCES}

1. Mian AA, Rafiei A, Haberbosch I, Zeifman A, Titov I, Stroylov V, et al. PF-114, a potent and selective inhibitor of native and mutated BCR/ABL is active against Philadelphia chromosome- positive $(\mathrm{Ph}+)$ leukemias harboring the T315I mutation. Leukemia 2015;29:1104-14.

2. Obr A, Roselova P, Grebenova D, Kuzelova K. Real-time analysis of imatinib-and dasatinib-induced effects on chronic myelogenous leukemia cell interaction with fibronectin. PloS One 2014;9:e107367.

3. Steinberg M. Dasatinib: a tyrosine kinase inhibitor for the treatment of chronic myelogenous leukemia and philadelphia chromosome-positive acute lymphoblastic leukemia. Clin Ther 2007;29:2289-308.

4. Latagliata R, Breccia M, Fava C, Stagno F, Tiribelli M, Luciano L, et al. Incidence, risk factors and management of pleural effusions during dasatinib treatment in unselected elderly patients with chronic myelogenous leukaemia. Hematol Oncol 2013;31:103-9.

5. Bertrand N, Wu J, Xu X, Kamaly N, Farokhzad OC. Cancer nanotechnology: the impact of passive and active targeting in the era of modern cancer biology. Adv Drug Deliv Rev 2014;66:2-5.

6. Jain RK, Stylianopoulos T. Delivering nanomedicine to solid tumors. Nat Rev Clin Oncol 2010;7:653.

7. Muddineti OS, Ghosh B, Biswas S. Current trends in using polymer-coated gold nanoparticles for cancer therapy. Int J Pharm 2015;484:252-67.

8. Cobley CM, Chen J, Cho EC, Wang LV, Xia Y. Gold nanostructures: a class of multifunctional materials for biomedical applications. Chem Soc Rev 2011;40:44-56.

9. Dreaden EC, Mackey MA, Huang X, Kang B, El-Sayed MA. Beating cancer in multiple ways using nanogold. Chem Soc Rev 2011;40:3391-404.

10. Kumar A, Zhang X, Liang XJ. Gold nanoparticles: emerging paradigm for targeted drug delivery system. Biotechnol Adv 2013;31:593-606.

11. Vigderman L, Zubarev ER. Therapeutic platforms based on gold nanoparticles and their covalent conjugates with drug molecules. Adv Drug Delivery Rev 2013;65:663-76.

12. Birla SS, Tiwari VV, Gade AK, Ingle AP, Yadav AP, Rai MK. Fabrication of silver nanoparticles by Phoma glomerata and its combined effect against Escherichia coli, pseudomonas aeruginosa and staphylococcus aureus. Lett Appl Microbiol 2009;48:173-9.

13. Rai M, Yadav A, Gade A. CRC 675-current trends in phytosynthesis of metal nanoparticles. Crit Rev Biotechnol 2008;28:277-84.

14. Singh P, Kim YJ, Zhang D, Yang DC. Biological synthesis of nanoparticles from plants and microorganisms. Trends Biotechnol 2016;34:588-99.

15. Xue M, Hu S, Lu Y, Zhang Y, Jiang X, An S, et al. Development of chitosan nanoparticles as drug delivery system for a prototype capsid inhibitor. Int J Pharm 2015;495:771-82.

16. Agnihotri SA, Mallikarjuna NN, Aminabhavi TM. Recent advances on chitosan-based micro-and nanoparticles in drug delivery. J Controlled Release 2004;100:5-28.

17. Boisselier E, Astruc D. Gold nanoparticles in nanomedicine: preparations, imaging, diagnostics, therapies and toxicity. Chem Soc Rev 2009;38:1759-82. 
18. Ehlerding EB, Chen F, Cai W. Biodegradable and renal clearable inorganic nanoparticles. Adv Sci 2016;3:1500223.

19. Sharma S, Parmar A, Kori S, Sandhir R. PLGA-based nanoparticles: a new paradigm in biomedical applications. Trends Anal Chem 2016;80:30-40.

20. Dhat S, Pund S, Kokare C, Sharma P, Shrivastava B. Risk management and statistical multivariate analysis approach for design and optimization of satranidazole nanoparticles. Eur J Pharm Sci 2017;96:273-83.

21. Garg NK, Sharma G, Singh B, Nirbhavane P, Tyagi RK, Shukla R, et al. Quality by Design (QbD)-enabled development of aceclofenac loaded-nano structured lipid carriers (NLCs): an improved dermatokinetic profile for the inflammatory disorder (s). Int J Pharm 2017;517:413-31.

22. Lawrence XY, Amidon G, Khan MA, Hoag SW, Polli J, Raju GK, et al. Understanding pharmaceutical quality by design. AAPS J 2014;16:771-83.

23. Sangshetti JN, Deshpande M, Zaheer Z, Shinde DB, Arote R. Quality by design approach: regulatory need. Arab J Chem 2017;10:S3412-25.

24. Upadhyay M, Adena SK, Vardhan H, Pandey S, Mishra B. Development and optimization of locust bean gum and sodium alginate interpenetrating polymeric network of capecitabine. Drug Dev Ind Pharm 2018;44:511-21.

25. Clogston JD, Patri AK. Zeta potential measurement. In: Scott E, McNeil. editor. Characterization of nanoparticles intended for drug delivery. $1^{\text {st }}$ ed. New Jersey: Humana Press; 2011. p. 63-70.

26. Govender T, Stolnik S, Garnett MC, Illum L, Davis SS. PLGA nanoparticles prepared by nanoprecipitation: drug loading and release studies of a water-soluble drug. J Controlled Release 1999;57:171-85.

27. Upadhyay M, Adena SK, Vardhan H, Yadav SK, Mishra B. Locust bean gum and sodium alginate-based interpenetrating polymeric network microbeads encapsulating capecitabine: improved pharmacokinetics, cytotoxicity and in vivo antitumor activity. Mater Sci Eng C 2019;104:109958.

28. Vardhan H, Mittal P, Adena SK, Upadhyay M, Mishra B. Development of long-circulating docetaxel loaded poly (3hydroxybutyrate-co-3-hydroxyvalerate) nanoparticles: optimization, pharmacokinetic, cytotoxicity and in vivo assessments. Int J Biol Macromol 2017;103:791-801.

29. Soshnikova V, Kim YJ, Singh P, Huo Y, Markus J, Ahn S, et al. Cardamom fruits as a green resource for facile synthesis of gold and silver nanoparticles and their biological applications. Artif Cells Nanomed Biotechnol 2018;46:108-17.

30. Engelbrekt C, SØrensen KH, Zhang J, Welinder AC, Jensen PS, Ulstrup J. Green synthesis of gold nanoparticles with starchglucose and application in bioelectrochemistry. J Mater Chem 2009;19:7839-47.

31. Rao K, Imran M, Jabri T, Ali I, Perveen S, Ahmed S, et al. Gum tragacanth stabilized green gold nanoparticles as cargos for naringin loading: a morphological investigation through AFM. Carbohydr Polym 2017;174:243-52.

32. Vardhan H, Mittal P, Adena SK, Mishra B. Long-circulating polyhydroxybutyrate-co-hydroxyvalerate nanoparticles for tumor-targeted docetaxel delivery: formulation, optimization and in vitro characterization. Eur J Pharm Sci 2017;99:85-94.

33. Soni N, Soni N, Pandey H, Maheshwari R, Kesharwani P, Tekade RK. Augmented delivery of gemcitabine in lung cancer cells exploring mannose anchored solid lipid nanoparticles. J Colloid Interface Sci 2016;481:107-16.

34. Adena SK, Upadhyay M, Vardhan H, Mishra B. Development, optimization, and in vitro characterization of dasatinib-loaded PEG functionalized chitosan capped gold nanoparticles using Box-Behnken experimental design. Drug Dev Ind Pharm 2018;44:493-501.
35. Peppas NA. Analysis of fickian and non-fickian drug release from polymers. Pharm Acta Helv 1985;60:110-1.

36. Fornaguera C, Caldero G, Mitjans M, Vinardell MP, Solans C, Vauthier C. Interactions of PLGA nanoparticles with blood components: protein adsorption, coagulation, activation of the complement system and hemolysis studies. Nanoscale 2015;7:6045-58.

37. Alkilany AM, Murphy CJ. Toxicity and cellular uptake of gold nanoparticles: what we have learned so far? J Nanopart Res 2010;12:2313-33.

38. Vardhan H, Mittal P, Adena SK, Upadhyay M, Yadav SK, Mishra B. Process optimization and in vivo performance of docetaxel loaded PHBV-TPGS therapeutic vesicles: a synergistic approach. Int J Biol Macromol 2018;108:729-43.

39. Skehan P, Storeng R, Scudiero D, Monks A, McMahon J, Vistica $D$, et al. New colorimetric cytotoxicity assay for anticancer-drug screening. J Natl Cancer Inst 1990;82:1107-12.

40. Vichai V, Kirtikara K. Sulforhodamine B colorimetric assay for cytotoxicity screening. Nat Protoc 2006;1:1112.

41. Rieger AM, Nelson KL, Konowalchuk JD, Barreda DR. Modified annexin $\mathrm{V} /$ propidium iodide apoptosis assay for accurate assessment of cell death. J Vis Exp 2011;50:2597.

42. Pund S, Shete Y, Jagadale S. Multivariate analysis of physicochemical characteristics of lipid-based nanoemulsifying cilostazol-quality by design. Colloids Surf B 2014;115:29-36.

43. Bhokare SG, Marathe RP. Rosuvastatin calcium loaded chitosan nanoparticles: preparation evaluation and in vitro release studies. Int J Appl Pharm 2020;7:95-102.

44. Viswanadh MK, Vikas, Jha A, Reddy Adena SK, Mehata AK, Priya $\mathrm{V}$, et al. Formulation and in vivo efficacy study of cetuximab decorated targeted bioadhesive nanomedicine for non-smallcell lung cancer therapy. Nanomedicine 2020;15:2345-67.

45. Tahir K, Nazir S, Li B, Khan AU, Khan ZU, Gong PY, et al. Nerium oleander leaves extract mediated synthesis of gold nanoparticles and its antioxidant activity. Mater Lett 2015;156:198-201.

46. Pertiwi RD, Suwaldi EP, Martien R. Bio-nanoparticles: green synthesis of gold nanoparticles and assessment of biological evaluation. Int J Appl Pharm 2019;11:133-8.

47. Devi L, Gupta R, Jain SK, Singh S, Kesharwani P. Synthesis, characterization and in vitro assessment of colloidal gold nanoparticles of Gemcitabine with natural polysaccharides for treatment of breast cancer. J Drug Delivery 2020;56:101565.

48. Rao K, Imran M, Jabri T, Ali I, Perveen S, Ahmed S, et al. Gum tragacanth stabilized green gold nanoparticles as cargos for naringin loading: a morphological investigation through AFM. Carbohydr Polym 2017;174:243-52.

49. Niza E, Noblejas Lopez MD, Bravo I, Nieto Jimenez C, Castro Osma JA, Canales Vazquez J, et al. Trastuzumab-targeted biodegradable nanoparticles for enhanced delivery of dasatinib in HER2+metastasic breast cancer. Nanomaterials 2019;9:1793.

50. Chen X, Hu Z, Zhou L, Zhang F, Wan J, Wang H. Self-assembling a natural small molecular inhibitor that shows aggregationinduced emission and potentiates antitumor efficacy. Nanoscale Horiz 2020;6:33-42.

51. Mandal S, Bakeine GJ, Krol S, Ferrari C, Clerici AM, Zonta C, et al. Design, development and characterization of multifunctionalized gold nanoparticles for biodetection and targeted boron delivery in BNCT applications. Appl Radiat Isot 2011;69:1692-7.

52. Thapliyal A, Chandra A. Antibacterial and anticancer potential of silver nanoparticles synthesized using gallic acid in bentonite/starch bio-nano composites. Int J Appl Pharm 2018;10:178-89.

53. Derbala SA, Elkady ME, Elbanhawy RA, AF AA. Antiproliferative and antioxidant effects of eruca sativa (jarjeer) leaves extract on carcinoma of women's breast. Asian J Pharm Clin Res 2021;9:89-92. 